\title{
1 A gatekeeping role of ESR2 to maintain the primordial follicle reserve
}

3 V. Praveen Chakravarthi ${ }^{1}$, Subhra Ghosh ${ }^{1}$, Katherine F. Roby ${ }^{2}$, Michael W. Wolfe ${ }^{3}$, and M. A. $4 \quad$ Karim Rumi ${ }^{1 *}$

$6 \quad{ }^{1}$ Deprartment of Pathology and Laboratory Medicine, ${ }^{2}$ Department of Anatomy and Cell Biology,

$7 \quad{ }^{2}$ Department of Molecular and Integrative Physiology, University of Kansas Medical Center,

8 Kansas City, Kansas, USA

9

10 Short title: ESR2 regulates primordial follicle activation

11

12 Keywords: Estrogen receptor $\beta$ (ESR2), mutant rat models, primordial follicle activation,

13 premature ovarian insufficiency, and activation of the AKT and mTOR pathways.

14

15 * Correspondence: M. A. Karim Rumi, mrumi@kumc.edu

16

18 Grants or fellowships support: This study was supported in part by the School of Medicine

19 Bridging grant from the University of Kansas Medical Center.

23 Disclosure: The authors do not have any conflict of interest. 


\section{Abstract}

25 Over the entire reproductive lifespan in mammals, a fixed number of primordial follicles serve as

26 the source of mature oocytes. Uncontrolled and excessive activation of primordial follicles can

27 lead to depletion of the ovarian reserve. We observed that disruption of ESR2-signaling results in

28 increased activation of primordial follicles in Esr2-null (Esr2-/-) rats. However, follicle assembly

29 was unaffected, and the total number of follicles remained comparable between neonatal wildtype

30 and Esr2-/- ovaries. While the activated follicle counts were increased in Esr2-/- ovary, the number

31 of primordial follicles were markedly decreased. Excessive recruitment of primordial follicles led

32 to premature ovarian senescence in Esr2-/- rats and was associated with reduced levels of serum

$33 \mathrm{AMH}$ and estradiol. Disruption of ESR2-signaling through administration of a selective antagonist

34 (PHTPP) increased the number of activated follicles in wildtype rats, whereas a selective agonist

35 (DPN) decreased follicle activation. In contrast, primordial follicle activation was not increased in

36 the absence of ESR1 indicating that the regulation of primordial follicle activation is ESR2-

37 specific. Follicle activation was also increased in Esr2-mutants lacking the DNA-binding domain,

38 suggesting a role for the canonical transcriptional activation function. Both primordial and

39 activated follicles express ESR2 suggesting a direct regulatory role for ESR2 within these follicles.

40 We also detected that loss of ESR2 augmented the activation of AKT, ERK and mTOR pathways.

41 Our results indicate that the lack of ESR2 upregulated both granulosa and oocyte factors, which

42 can facilitate AKT and mTOR activation in Esr2-/- ovaries leading to increased activation of 43 primordial follicles. 


\section{Introduction}

48 Primordial follicles generated during early life serve as the source of fertilizable oocytes in

49 mammals throughout the reproductive lifespan (1). A limited number of primordial follicles are

50 steadily recruited into the growing pool while the remaining are maintained in the dormant state

51 (2,3). The female reproductive life comes to an end when folliculogenesis ceases due to the loss

52 of primordial follicles $(4,5)$. Regulation of the dormancy and selective activation of primordial

53 follicles plays an important role in determining the reproductive lifespan in females (6). An

54 uncontrolled and increased activation of primordial follicles leads to excessive depletion of the

55 ovarian reserve and development of premature ovarian insufficiency (POI), which causes

56 infertility and estrogen deficiency in 1-2\% of all women of reproductive age (7-9). Pathological

57 conditions as well as certain therapeutic procedures that deplete the follicle reserve can also lead

58 to POI (10). Understanding the regulatory mechanisms of primordial follicle activation remains

59 critical for prevention or treatment of POI.

60 Activation of primordial follicles to primary follicles is a strictly regulated and steady

61 process. This activation is gonadotropin independent, but the exact molecular mechanisms of the

62 prolonged dormancy and selective recruitment of primordial follicles remain largely unclear (11).

63 Primordial follicles consist of dormant oocytes surrounded by a few flattened granulosa cells

64 (GCs). Activation of the primordial follicle is characterized by growth and differentiation of the

65 flattened GCs to cuboidal GCs as well as oocyte growth and deposition of the zona pellucida (ZP)

66 matrix by the oocytes (12-14). Signaling pathways in both GCs and oocytes have been found to

67 play important roles in primordial follicle activation (15). It has been suggested that the process

68 starts in the GCs, and the signals from GCs subsequently activating the oocytes in primordial

69 follicles $(16,17)$. Conversely, studies have also indicated that the activation process can be initiated 
70 in the oocytes of primordial follicles (14). Inhibitory factors secreted from the primordial follicles

71 or from activated follicles may also maintain the primordial follicles in a dormant state (18).

72 Previous studies with mutant mouse models have identified several growth factors and

73 cytokines involved in primordial follicle activation. While TGF- $\beta$ superfamily members such as

74 bone morphogenic proteins (BMPs) and growth and differentiation factors (GDFs) act as positive

75 regulators $(19,20), \mathrm{AMH}$ acts as a negative regulator of primordial follicle activation (21). Growth

76 factors unrelated to the TGF- $\beta$ superfamily including KITLG (22) and FGF2 (23), also enhance

77 the activation process. These growth factors and cytokines regulate key signaling pathways and

78 transcriptional regulators. Regulation of PI3 kinase (PI3K) and mTOR-signaling and the

79 downstream transcription factor FOXO3A are essential regulators of primordial follicle activation

80 (14,24). In addition to FOXO3A (25), several other transcription factors have been found to be

81 important for the regulation of follicle activation. FOXL2 (26) in primordial GCs as well as

82 SOHLH1 (27), SOHLH2 (28), NOBOX (29), LHX8 (27), GATA4, and GATA6 (30) in oocytes

83 carry out important regulatory functions. We recently observed that transcriptional regulation

84 mediated by estrogen receptor $\beta$ (ESR2) plays an essential role during primordial follicle

85 activation.

ESR2 is the predominant estrogen receptor in the ovary (31-34). ESR2 polymorphisms and

87 mutations in women have been linked to ovulatory dysfunctions, including complete ovarian

88 failure (35-38). Esr2-mutant mouse $(39,40)$ and rat models (41) suffer from defective follicle

89 development and failure of ovulation. Studies have suggested that estrogen signaling plays a role

90 in follicle assembly and early follicle development $(42,43)$, but a role of ESR2 in ovarian

91 primordial follicle activation has not yet been demonstrated. In this study, we observed that

92 disruption of ESR2 signaling markedly increases the activation of primordial follicles. Our 
93 findings suggest that the transcriptional regulatory function of ESR2 plays a gatekeeping role in

94 maintaining the primordial follicle reserve.

95

96 Materials and Methods

97 Animal models

98 Wildtype, Esr1-knockout, and Esr2-mutant Holtzman Sprague-Dawley (HSD) rats were included

99 in this study. The Esrl-knockout rat model was generated by the deletion of exon 3 in

100 the Esrl gene, which caused a frameshift and null mutation (Esr1-/-) (44). Esr2-mutant rat

101 models were generated by targeted deletion of the exon 3 or exon 4 in the Esr 2 gene (41). Exon 3

102 deletion caused a frameshift and null mutation (Esr2-/-), whereas exon 4 deletion resulted in a

103 mutant ESR2 protein lacking the DNA binding domain (DBD). Rats were screened for the

104 presence of mutations by PCR using tail-tip DNA samples (RED extract-N-Amp Tissue PCR Kit,

105 Sigma-Aldrich) as previously described (41,44). All procedures were performed in accordance

106 with the protocols approved by the University of Kansas Medical Center Animal Care and Use

107 Committee.

108

109 Gonadotropin treatment for follicle maturation

110 28-day-old Esr2-/- and age-matched wildtype female rats were used for the evaluation of 111 gonadotropin-induced ovarian follicle development. Synchronized follicle maturation was induced

112 by intraperitoneal injection of 30 IU PMSG (Lee Biosolutions, Maryland Heights, MO). 48h after

113 the PMSG injection, $30 \mathrm{IU}$ of hCG (Lee Biosolutions) was injected intraperitoneally. 4h after hCG

114 injection, ovaries were collected and processed for histological examination, or cumulus-oocyte

115 complexes (COCs) were harvested by needle-puncture of the large antral follicles under 
116 microscopic examination as described previously (45). Cumulus cells were mechanically detached

117 from the COCs by repeated pipetting, and the oocytes were counted under microscopic

118 examination.

120 Treatment with selective ESR2-antagonist and -agonist

121 A selective ESR2 antagonist PHTPP (46) or a selective ESR2 agonist DPN (47) (Cayman

122 Chemical, Ann Arbor, MI) was dissolved in DMSO $(5 \mu \mathrm{g} / \mu \mathrm{l})$. $2 \mu \mathrm{l}$ of PHTPP (10 $\mu \mathrm{g})$ or $2 \mu \mathrm{l}$ of

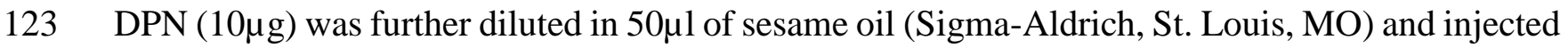

124 subcutaneously into wildtype rats from postnatal day (PND) 5 to 15 . Rats were sacrificed on

125 PND16, and the ovaries were processed either for histological examination or for total RNA and

126 protein analyses.

128 Blood collection and hormone assays

129 Blood samples were collected by cardiac puncture at sacrifice. After clotting at room temperature,

130 serum was separated by centrifugation and frozen at -80C. Serum AMH level was determined by

131 an enzyme immunoassay (AL-113, Ansh Labs, Webster, TX). Total serum 17ß-estradiol (E2) and

132 serum progesterone (P4) levels were measured by RIA as described previously (48).

134 Total follicle counting in the serial sections of whole ovary

135 Ovaries were collected and fixed in $4 \%$ formaldehyde overnight, processed, and embedded in 136 paraffin following standard procedures (49-51). Whole ovaries were serially sectioned at $6 \mu \mathrm{M}$ 137 thickness and stained with hematoxylin and eosin (H\&E) (49-51). The stages of follicle 138 development including primordial, primary, secondary, early antral, and antral were determined 
139 as described previously (52). The primordial follicles were recognized as small oocytes surrounded

140 by a few flattened GCs, the primary follicles contained larger oocytes surrounded by a single layer

141 of cuboidal GCs, and the oocytes in the secondary follicles were surrounded by multiple layers of

142 GCs. The tertiary follicles were categorized into early antral and antral follicles based on

143 appearance and extent of the cavities within the follicles (Figure 1D). Atretic follicles were

144 recognized by pyknotic GCs surrounding degenerated oocytes. The follicles were counted on every

145 fifth section under light microscopy $(49,50)$. To avoid counting the same follicle more than once,

146 primordial and primary follicles were counted if they exhibited a nucleus, whereas the secondary,

147 early antral, and antral follicles were counted only in presence of a nuclei with prominent nucleoli

148 (49-51). The counts were multiplied by 5 to obtain the total count of follicles in the whole ovary.

\section{Protein extraction and western blotting}

151 Ovaries were collected on PND 4, 6, and 8, and total protein was extracted from the ovaries using 152 1X SDS lysis buffer (62.5mMTris-HCl pH 6.8, 2\% SDS, 42 mM dithiothreitol, 10\% glycerol, and $153 \quad 0.01 \%$ bromophenol blue), containing protease and phosphatase inhibitors (Cell Signaling 154 Technologies, Danvers, MA). Ovarian lysates were sonicated to shear DNA and reduce viscosity, 155 heat denatured, and separated on a 4-20\% SDS-PAGE. Electrophoresed proteins were transferred 156 from the gel to PVDF membranes, blocked with 5\% skim milk in TBST (1xTBS buffer containing $1570.1 \%$ Tween-20), and incubated for $1 \mathrm{~h}$ at room temperature with specific primary antibodies 158 (Table 1) at the appropriate dilution in blocking solution. After removing the unbound primary 159 antibody solution, membranes were washed with TBST, blocked, and incubated with peroxidase160 conjugated anti-mouse, or anti-rabbit secondary antibodies (Jackson Immunoresearch, West 
161 Grove, PA) at a dilution of 1:25,000 to 50,000, and the immunoreactivity signals were visualized

162 with Luminata Crescendo HRP substrate (Millipore Sigma, Burlington, MA).

\section{RNA extraction and RT-qPCR}

165 Total RNA was extracted from the PND8 ovaries using TRI Reagent (Sigma-Aldrich). 1000ng of

166 total RNA from each sample was used for the preparation of cDNA using High-Capacity cDNA

167 Reverse Transcription (RT) Kits (Applied Biosystems, Foster City, CA). The cDNA was diluted

$168 \quad 1: 50$ in $10 \mathrm{mM}$ Tris- $\mathrm{HCl}(\mathrm{pH} 7.4)$ and $2.5 \mu \mathrm{l}$ of the diluted cDNA was used in a $10 \mu l$ quantitative

169 PCR (qPCR) reaction mixture containing Applied Biosystems Power SYBR Green PCR Master

170 Mix (Thermo Fisher Scientific). Amplification and fluorescence detection of RT-qPCR were

171 carried out on Applied Biosystems QuantStudio Flex 7 Real Time PCR System (Thermo Fisher

172 Scientific). The $\Delta \Delta \mathrm{CT}$ method was used for relative quantification of target mRNA expression

173 and normalized to Rn18s (18S rRNA) level. A list of qPCR primers is shown in Table 2.

174

\section{Isolation of primordial and primary follicles}

176 Approximately $100 \mathrm{mg}$ of minced PND12 rat ovary tissue was digested in $1 \mathrm{ml}$ of digestion

177 medium [199 media containing $0.08 \mathrm{mg} / \mathrm{ml}$ of liberase with medium-concentration of thermolysin

178 (Roche Diagnostics GmbH, Mannheim, Germany) supplemented with 5U/ml of DNase I and 1\%

179 BSA (Thermo Fisher Scientific, Waltham, MA)]. The digestion mix was agitated on an orbital

180 shaker (Disruptor Genie, Scientific Industries, Bohemia, NY) at $1500 \mathrm{rpm}$ for $30 \mathrm{~min}$ at room

181 temperature. The enzymatic reaction was stopped by addition of 10\% FBS. Digested ovary tissues

182 were passed through a $70 \mu \mathrm{M}$ cell strainer (Thermo Fisher Scientific) to remove the secondary, and

183 large follicles as well as tissue aggregates. The filtrate containing the small follicles and cellular 
184 components was filtered again through a $35 \mu \mathrm{M}$ cell strainer (BD Falcon, Franklin Lakes, NJ). The

$18535 \mu \mathrm{M}$ strainer was reverse eluted with medium 199 to isolate the primary follicles and the filtrate

186 was subjected to sieving through a $10 \mu \mathrm{M}$ cell strainer (PluriSelect USA, Gillespie Way, CA) to

187 separate the primordial follicles from other cellular components. Finally, the $10 \mu \mathrm{M}$ cell strainer

188 was reverse eluted to isolate the primordial follicles. Unwanted cellular components were removed

189 from the desired follicles under microscopic examination before proceeding to RNA isolation.

191 Gene expression analyses in primordial and primary follicles

192 We used 200-250 primordial follicles and 100-150 primary follicles for cDNA synthesis using the

193 Message Booster cDNA synthesis kit (Lucigen, Palo Alto, CA). Direct cDNA and subsequent

194 cRNA syntheses were performed by following the manufacturer's instruction. In vitro synthesized

195 cRNA was purified by using Monarch RNA cleanup kit (New England Biolabs, Ipswich, MA) and

196 subjected to first strand, and subsequent second strand cDNA synthesis using the regents provided

197 in the Message Booster cDNA synthesis kit. The cDNA was diluted 1:10 in 10mM Tris- $\mathrm{HCl}(\mathrm{pH}$

198 7.4) and $2.5 \mu \mathrm{l}$ of the diluted cDNA was used in $10 \mu \mathrm{l}$ qPCR reaction as described above. The

199 relative quantification of target mRNA expression was calculated by normalizing the data with

$200 \quad A c t b$ expression.

201

202 Statistical analyses

203 Follicle counting was performed on more than 3 individual rats of the same genotype at each time

204 point. Gene expression analyses were performed on at least 6 individual rats. The experimental

205 results are expressed as mean \pm SE. Statistical comparisons between two means were determined

206 with Student's t-test while comparisons among multiple means were evaluated with ANOVA 
207 followed by Duncan post hoc test. $P$ values $\leq 0.05$ were considered as significant level of

208 difference. All statistical calculations were done with SPSS 22 (IBM, Armonk, NY).

\section{$210 \quad$ Results}

\section{Increased number of antral follicles in Esr2-/- rats}

212 Exogenous gonadotropins were administered to PND28 prepubertal rats to induce follicle 213 maturation. Compared to the wildtype, Esr2-/- ovaries exhibited a larger number of antral follicles

214 4h after hCG administration into PMSG primed rats (Figure 1A, B). At this time point, cumulus-

215 oocyte complexes (COCs) were isolated from the gonadotropin-stimulated ovaries by needle

216 puncture, and cumulus cells were mechanically removed to count the oocytes. Oocyte counts were

217 significantly higher in Esr2-/- rats (Figure 1C). To compare the extent of follicle maturation

218 between wildtype and Esr2-/- rats, total follicle counting was performed in serial sections of 219 gonadotropin-stimulated ovaries. While the number of primordial follicles were decreased, the 220 number of primary, secondary, and antral follicles were markedly increased in the ovaries of Esr2-

221 /- rats (Figure 1E-G). We further investigated the status of follicle activation in Esr2 mutant rats 222 prior to gonadotropin treatment.

\section{Loss of ESR2 leads to the activation of primordial follicles}

225 We detected an increase in the recruitment of primordial follicles to the growing pool in PND28 226 Esr2-/- rat ovaries in the absence of any gonadotropin treatment (Figure $\mathbf{2 A - C}, \mathbf{J}, \mathbf{M}$ ). To 227 determine the postnatal period during which increased follicle activation becomes evident, further 228 ovarian follicle counting was performed on PND16 and PND8. A decrease in primordial follicle 229 counts with a corresponding increase in the number of activated follicles was detected in both 
PND16 (Figure 2D-F, K, N) and PND8 (Figure 2G-I, L, O) Esr2-/- ovaries. At all-time points,

231 increased primordial follicle activation was found to be approximately twofold greater in Esr2-/-

232 rats compared to wildtype. However, there was an increased number of atretic follicles in Esr2-/-

233 ovaries (Figure 2B, E, H).

\section{Loss of ESR2 results in premature ovarian senescence}

236 We observed that total follicle counts in PND8 ovaries were similar between the wildtype and 237 Esr2-/- rats; however, the primordial follicle counts were decreased by $\sim 35 \%$ in Esr2-/- ovaries

238 (Figure 3A). Further analyses of follicle count in 4wk, 12wk, and 24wk old Esr2-/- and age239 matched wildtype rats revealed a sharp decline in primordial follicles in Esr2-/- ovaries (Figure

240 3B-H). Moreover, this decline in ovarian follicle numbers was associated with a significantly 241 lower levels of serum estradiol and AMH in 24 wk old Esr2-/- rats. (Figure 3I, J).

\section{Regulation of primordial follicle activation is ESR2-dependent}

244 Similar to Esr2-/- rats, an increased activation of primordial follicles was also observed in rats 245 carrying a homozygous mutation in the DBD of Esr2 (Figure 4A, B, G, I, J). The number of 246 growing follicles was markedly increased while the number of primordial follicles decreased.

247 However, this altered recruitment of primordial follicles was not detected in Esr 1-/- rat ovaries

248 (Figure 4A, C, G, I, K). In addition, treatment of wildtype rats with a selective ESR2-antagonist 249 (PHTPP) resulted in increased activation of primordial follicles (Figure 4D, E, H, L, M). In 250 contrast, treatment of wildtype rats with a selective ESR2-agonist (DPN) increased the proportion 251 of follicles in the primordial state (Figure 4D, F, H, L, N). 


\section{Disruption of ESR2 resulted in activation of the AKT and mTOR pathways}

254 We detected abundant expression of ESR2 mRNA and protein in PND4, 6 and 8 ovaries (Figure

5 A, B). Expression of Esr2 was also evident in primordial and primary follicles isolated from

256 wildtype rat ovaries (Figure 5 C-E). Previous studies have shown that primordial follicle

257 activation is regulated by the PI3K-PTEN-AKT and the mTORC1-TSC1/2-P70S6K signaling

258 pathways $(14,53)$. We therefore assessed the activation status of selected signaling molecules

259 within these pathways in PND8 Esr2-/- ovaries and compared with that of wildtype. We observed

260 increased activation of pAKT (T308 and S473) (Figure 5F-I), pERK1/2 (T202/Y204) (Figure

261 5L-M), mTORC1 (S2448) (Figure 5P-Q), and the downstream targets p70S6K (T389) (Figure

262 5R-S) and pRPS6 (S240/244) (Figure 5T-U). However, no significant alteration in PTEN levels

263 (Figure 5J-K) or pTSC2 (T1462) phosphorylation (Figure 5N-O) was observed in Esr2-/-

264 ovaries.

Loss of ESR2 increased expression of factors upstream of AKT and mTOR signaling

267 ESR2 is a transcriptional regulator and we have determined that the DB- dependent transcriptional

268 activation function of ESR2 is required for regulation of primordial follicle activation (Figure 4).

269 Therefore, we assessed the expression of factors that are known to activate the AKT and mTOR

270 pathways and increase primordial follicle activation. Upstream regulators of AKT signaling

271 including Kitlg, Kit, and Igfl (Figure 6A-C) as well as Adcyaplrl (Figure 6F) were upregulated

272 in PND8 Esr2-/- ovaries. Oocyte derived TGF $\beta$ family members including Bmp4, Bmp15, and

273 Gdf9 were also significantly upregulated in Esr2-/- ovaries (Figure 6G-I). In addition,

274 transcriptional regulators Gata4 (Figure 6N), and Npm2 (Figure 60) were markedly upregulated

275 in Esr2-/- ovaries. However, expression of Fgf2 (Figure 6D), Ntrk2 (Figure 6E), Amh (Figure 
6J), Bdnf (Figure 6K), Nobox (Figure 6L), and Sohlh1(Figure 6M) was similar between Esr2-/-

277 and wildtype ovaries.

\section{Discussion}

280 During the isolation of oocytes from the gonadotropin stimulated PND28 ovaries, we were 281 surprised by a threefold higher yield in Esr2-/- rats. This appears to be due to the presence of a 282 large number of activated follicles in Esr2-/- ovaries that were able to respond to gonadotropin 283 treatment. This increased number of activated follicles was associated with a decreased number of 284 primordial follicles suggesting excessive recruitment of primordial follicles. It is well known that 285 activation of primordial follicles is independent of gonadotropin signaling (11). Thus, increased 286 activation of primordial follicles observed in Esr2-/- rats was presumably independent of 287 gonadotropin stimulation. It was confirmed by an increased activation of primordial follicles in 288 PND8 and PND16 Esr2-/- ovaries and these findings suggest involvement of an intraovarian 289 mechanism, which was defective during the early ovarian development due to the lack of ESR2. 
In spite of normal follicle assembly and the establishment of the primordial follicle pool, excessive activation resulted in premature depletion of the primordial follicles in Esr2-/- rats. By 24 weeks of age, Esr2-/- rats contained less than $15 \%$ of the primordial follicles observed in wildtype ovaries (Fig 3B). As expected, the rapid decline in the follicle reserve led to premature ovarian senescence associated with a low AMH and estradiol level. AMH is an established indicator of follicle reserve and the serum level correlates well with the fertility potential $(56,57)$. As polymorphisms or mutations can affect ESR2 function (35-38) and some endocrine disruptors can act as ESR2 agonists or antagonists (58-61), ESR2 regulation of primordial follicle activation can have a significant impact on women's health and fertility. secretion associated with a failure in follicle development and ovulation (39-41). Administration of exogenous gonadotropins fails to induce normal follicle maturation and ovulation in these mutant animals suggesting that disruption of estrogen signaling results in a primary ovarian defect

311 (39-41). Increased activation of primordial follicles in Esr2-/- rats represents such a primary 312 intraovarian defect. Absence of any increased activation of primordial follicles in Esrl-/- rats

313 indicates that the regulatory mechanism is specific to ESR2. Furthermore, complete loss of ESR2 314 or loss of the DBD of ESR2 led to a similar phenotype, highlighting the requirement of the 315 canonical transcriptional function of ESR2 in maintaining the primordial follicle reserve. In line 316 with these observations, manipulation of ESR2 signaling by administration of selective antagonist 317 or agonist into wildtype rats demonstrated that ESR2 plays a gatekeeping role during primordial 318 follicle recruitment. Thus, transcriptional activation of ESR2 with selective ligands may 319 prospectively increase the reproductive longevity in females. Selective ESR2-antagonists may also 320 prove effective for in vitro activation (IVA) of primordial follicles. 
It has been previously demonstrated that increased activation of AKT leads to accelerated

322 activation of primordial follicles and depletion of the follicle reserve $(62,63)$. Thus, increased

323 activation of AKT in Esr2-/- rats may be related to the increased activation of primordial follicles.

324 Esr2-/- rat ovaries also demonstrated increased activation of the mTOR and ERK pathways.

325 Activated AKT can activate the mTOR pathway, which can also promote primordial follicle

326 activation $(14,53)$. A recent study has shown that ERK activation can induce primordial follicle

327 activation through the KIT-mTOR signaling (64). Due to the complex architecture of the ovary

328 (containing follicles at different stages and extrafollicular cells), it is difficult to discern whether

329 these changes in signaling pathways are strictly due to accelerated primordial follicle development

330 or reflect changes within primordial follicles.

331 While screening for candidate genes that may act as upstream regulators of the PI3K-AKT

332 pathway, we identified upregulated expression of several growth factors and cytokines in Esr2-/-

333 ovaries (Figure 6,7). Among these growth factors, KITLG and IGF1 can activate receptor tyrosine

334 kinases on oocytes and induce activation of primordial follicles (22,65-67). The other upregulated

335 factors are oocyte-derived TGF $\beta$ family members including BMP4, BMP15, and GDF9, which are

336 known to stimulate follicle activation $(19,20)$. While GDF9 and BMP15 can activate the mTOR

337 pathway (68), BMP signaling can also activate the ERK pathway through TAK1 (69). In addition

338 to these factors, upregulation of ADCYAP1R1, and NPM2 were prominent in PND8 Esr2-/- rat

339 ovaries, which might have also contributed to the activation of the PI3K-AKT pathway. NPM2 is

340 a transcriptional regulator in oocytes (70), which was found to activate the AKT $(71,72)$, mTOR,

341 and ERK pathways in other cells (73). While ADCY3 is expressed in oocytes, its receptor

342 ADCYAP1R1 is expressed in GCs; ADCY3 signaling generates cAMP, which in turn can activate 
343 the AKT pathway (74). As stated previously, these factors may regulate follicle activation but how

344 ESR2 is implicating them in the ovary is currently unclear.

345 In this study, we demonstrate a novel role of ESR2 in maintaining the primordial follicle

346 reserve. Excessive activation of primordial follicles leads to premature loss of the follicle reserve.

347 Our findings suggest that ESR2 is involved in downregulating the expression of ovarian-derived

348 factors, which function to activate key signaling pathways that induce primordial follicle

349 activation. Loss of ESR2 upregulates these factors and results in an increased activation of the

350 signaling pathways that promotes primordial follicle activation.

352 Acknowledgments: We acknowledge the financial support from the KUMC School of Medicine

353 (SOM Bridging Grants) for completion of this research.

FIGURE LEGENDS

357 Figure 1. Gonadotropin stimulation resulted in an increased number of antral follicles in Esr2-/-

358 ovaries. Wildtype (WT) and Esr2-/- rats were treated with exogenous gonadotropins on PND28.

359 48h after PMSG (30IU) injection, rats were administered with hCG (30IU). 4h after the hCG

360 injection, ovaries were collected and processed for histological examination or collection of COCs.

361 Histological examination demonstrated the presence of ovarian follicles at different stages of

362 development in WT rats (A). In contrast, Esr2-/- rat ovaries showed an increased number of antral

363 follicles (B). Cumulus cells were detached from the oocytes by mechanical pipetting before

364 counting under microscope. Oocyte yield was about three-fold higher in Esr2-/- rat ovaries (C).

365 Serially sectioned whole ovaries were stained with H\&E, and follicles at different stages were 
counted in WT and Esr2-/- rats (D). Follicle counting demonstrated a decreased number of

367 primordial follicles and an increased number of activated follicles in Esr2-/- rats (E-G). Data

368 shown as mean $\pm \mathrm{SE}, \mathrm{n} \geq 3 .{ }^{*} P \leq 0.05$.

Figure 2. Increased activation of primordial follicles in Esr2-/- rat ovaries. Follicle counting in

371 PND28 (A-C, J, M), PND16 (D-F, K, N), and PND8 (G-I, L, O) wildtype (WT) and Esr2-/- rats showed increased activation of primordial follicles in Esr2-/- ovaries. Primordial follicle activation was near two-fold starting on PND8 (G-I, L, O). A greater number of atretic follicles were present within the pool of activated follicles at PND28 (A, B) PND16 (D, E) and PND8 (G, H) in Esr2-/ovaries. Data shown as mean $\pm \mathrm{SE}, \mathrm{n} \geq 3 .{ }^{*} P \leq 0.05$. Pd, Primordial follicle; Py, Primary follicle.

Figure 3. Premature ovarian senescence in Esr2-/- rats. The total number of follicles in PND8 ovaries were similar between wildtype (WT) and Esr2-/- rats; however, the number of primordial follicles were decreased in Esr2-/- ovaries (A). Follicle counts in 4wk, 12wk, and 24wk old WT and Esr2-/- rats revealed a sharp decline in the primordial follicle reserve in Esr2-/- ovaries (BH). 24wk old Esr2-/- rats showed a significantly lower level of serum estradiol and AMH compared to WT (I, J). Data shown as mean $\pm \mathrm{SE}, \mathrm{n} \geq 3$ (follicle counting) and $\mathrm{n} \geq 6$ (hormone assays), $* P \leq 0.05$. Pd, Primordial follicle; Py, Primary follicle.

Figure 4. Regulation of primordial follicle activation is ESR2-dependent. Deletion of the ESR2 observed in ovaries from Esr1-/- rats $(\mathbf{C}, \mathbf{G}, \mathbf{K})$. Administration of a selective ESR2 antagonist, 
treatment with an ESR2 agonist, DPN, suppressed the activation $(\mathbf{F}, \mathbf{H}, \mathbf{N})$. Data shown as mean $\pm \mathrm{SE}, \mathrm{n} \geq 3 .{ }^{*} P \leq 0.05$. Rel., Relative. Pd, Primordial follicle; Py, Primary follicle.

392 Figure 5. Activation of AKT, ERK, and mTOR signaling in Esr2-/- ovaries. Expression of ESR2

393 was detected in PND4, 6, and 8 rat ovaries using RT-qPCR (A) and western blotting (B).

394 Primordial (Pd) (C), and primary (Py) (D) follicles were isolated from rat ovaries by digestion with liberase followed by size fractionation with strainers. RT-qPCR analysis demonstrated Esr2 expression in both Pd and Py follicles (E). Western blot analyses of PND8 ovaries and quantification of signal intensities demonstrated a significant increase in AKT (F-I) and ERK1/2 (L-M) activation. This was associated with increased activation of mTORC1 (P-Q) and its targets P70S6K (R-S) and RPS6 (T-U). But no difference was observed in PTEN (J-K) and pTSC2 (NO) levels. Signal quantification data are presented as mean \pm SEM. $n \geq 6$. ${ }^{*} P \leq 0.05$. Rel., Relative.

Figure 6. Transcript levels of known activators of the AKT and mTOR pathways. RT-qPCR was performed on PND8 wildtype (WT) and Esr2-/- rat ovaries to analyze the expression of genes involved in activation of the AKT and mTOR pathways and activation of primordial follicles. While the expression of Kitlg (A), Kit (B), Igfl (C), Bmp15 (H) and Gdf9 (I) was moderately upregulated in Esr2-/- rat ovaries, Adcyaplrl(F), Bmp4 (G), Gata4 (N), and Npm2 (O) were highly upregulated. But no significant differences in the expression of Fgf2 (D), Ntrk2 (E), Amh $(\mathbf{J}), \operatorname{Bdnf}(\mathbf{K}), \operatorname{Nobox}(\mathbf{L})$ and $\operatorname{Sohlhl}(\mathbf{M})$ were observed between the WT and Esr2-/- rat ovaries. 
411 Figure 7. ESR2 signaling in the regulation of primordial follicle activation. Loss of ESR2 leads to

412 upregulation of both granulosa cell and oocyte derived factors that can activate the AKT, ERK and

413 mTOR pathways. Increased levels of KITLG, KIT and IGF1, as well as NPM2, and ADCYAP1R1

414 can activate the AKT pathway followed by the mTOR pathway. Moreover, upregulation of NPM2

415 and BMP4 can activate the ERK pathway. Activated AKT, ERK and mTOR pathways in

416 association with the transcriptional regulator GATA4 can promote the transition of primordial

417 follicles to primary follicles in Esr2-/- ovaries.

419 References

420 1. Zuckerman S. The Number of Oocytes in the Mature Ovary. Recent Prog Horm Res 1951;

$421 \quad 6: 63-109$

422 2. Peters H. The development of the mouse ovary from birth to maturity. Acta $423 \quad$ endocrinologica $1969 ; 62: 98-116$

424 3. Peters H, Levy E, Crone M. Deoxyribonucleic acid synthesis in oocytes of mouse embryos. $425 \quad$ Nature $1962 ; 195: 915-916$

426 4. Li L, Fu Y-c, Xu J-j, Lin X-h, Chen X-c, Zhang X-m, Luo L-l. Caloric restriction promotes 427 the reserve of follicle pool in adult female rats by inhibiting the activation of mammalian $428 \quad$ target of rapamycin signaling. Reprod Sci 2015; 22:60-67

429 5. Bristol-Gould SK, Kreeger PK, Selkirk CG, Kilen SM, Mayo KE, Shea LD, Woodruff TK. $430 \quad$ Fate of the initial follicle pool: empirical and mathematical evidence supporting its $431 \quad$ sufficiency for adult fertility. Developmental biology 2006; 298:149-154 
432 6. Adhikari D, Risal S, Liu K, Shen Y. Pharmacological inhibition of mTORC1 prevents 433 over-activation of the primordial follicle pool in response to elevated PI3K signaling. PloS $434 \quad$ one $2013 ; 8:$ e53810-e53810

435 7. De Vos M, Devroey P, Fauser BC. Primary ovarian insufficiency. Lancet (London, 436 England) 2010; 376:911-921

437 8. Coulam CB, Adamson SC, Annegers JF. Incidence of premature ovarian failure. Obstetrics $438 \quad$ and gynecology $1986 ; 67: 604-606$

439 9. Nelson LM. Clinical practice. Primary ovarian insufficiency. The New England journal of $440 \quad$ medicine $2009 ; 360: 606-614$

441 10. Jankowska K. Premature ovarian failure. Prz Menopauzalny 2017; 16:51-56

442 11. Hsueh AJW, Kawamura K, Cheng Y, Fauser BCJM. Intraovarian Control of Early $443 \quad$ Folliculogenesis. Endocrine reviews 2015; 36:1-24

444 12. Meredith S, Dudenhoeffer G, Jackson K. Classification of small type B/C follicles as 445 primordial follicles in mature rats. Journal of reproduction and fertility 2000; 119:43-48

446 13. Epifano O, Liang LF, Familari M, Moos MC, Jr., Dean J. Coordinate expression of the three zona pellucida genes during mouse oogenesis. Development (Cambridge, England)

449 14. Adhikari D, Liu K. Molecular mechanisms underlying the activation of mammalian primordial follicles. Endocrine reviews 2009; 30:438-464

451 15. Nilsson EE, Kezele P, Skinner MK. Leukemia inhibitory factor (LIF) promotes the primordial to primary follicle transition in rat ovaries. Molecular and cellular endocrinology 2002; 188:65-73 
454 16. Zhang H, Liu K. Cellular and molecular regulation of the activation of mammalian primordial follicles: somatic cells initiate follicle activation in adulthood. Human reproduction update $2015 ; 21: 779-786$

17. Zhang H, Risal S, Gorre N, Busayavalasa K, Li X, Shen Y, Bosbach B, Brannstrom M, Liu

18. Da Silva-Buttkus P, Marcelli G, Franks S, Stark J, Hardy K. Inferring biological mechanisms from spatial analysis: prediction of a local inhibitor in the ovary. Proceedings

19. Silva JR, van den Hurk R, van Tol HT, Roelen BA, Figueiredo JR. Expression of growth

21. Durlinger AL, Kramer P, Karels B, de Jong FH, Uilenbroek JT, Grootegoed JA, Themmen

20. Sun RZ, Lei L, Cheng L, Jin ZF, Zu SJ, Shan ZY, Wang ZD, Zhang JX, Liu ZH. Expression of GDF-9, BMP-15 and their receptors in mammalian ovary follicles. J Mol Histol 2010;

22. Parrott JA, Skinner MK. Kit-ligand/stem cell factor induces primordial follicle

23. Nilsson E, Parrott JA, Skinner MK. Basic fibroblast growth factor induces primordial follicle development and initiates folliculogenesis. Molecular and cellular endocrinology 2001; 175:123-130 
24. Adachi S, Yamada S, Takatsu Y, Matsui H, Kinoshita M, Takase K, Sugiura H, Ohtaki T, Matsumoto $\mathrm{H}$, Uenoyama $\mathrm{Y}$, Tsukamura $\mathrm{H}$, Inoue $\mathrm{K}$, Maeda $\mathrm{K}$. Involvement of anteroventral periventricular metastin/kisspeptin neurons in estrogen positive feedback action on luteinizing hormone release in female rats. The Journal of reproduction and development 2007; 53:367-378

25. Castrillon DH, Miao L, Kollipara R, Horner JW, DePinho RA. Suppression of ovarian follicle activation in mice by the transcription factor Foxo3a. Science (New York, NY) $2003 ; 301: 215-218$

26. Schmidt D, Ovitt CE, Anlag K, Fehsenfeld S, Gredsted L, Treier AC, Treier M. The murine winged-helix transcription factor Foxl2 is required for granulosa cell differentiation and ovary maintenance. Development (Cambridge, England) 2004; 131:933-942

27. Pangas SA, Choi Y, Ballow DJ, Zhao Y, Westphal H, Matzuk MM, Rajkovic A. Oogenesis requires germ cell-specific transcriptional regulators Sohlh1 and Lhx8. Proceedings of the National Academy of Sciences of the United States of America 2006; 103:8090-8095

28. Choi Y, Yuan D, Rajkovic A. Germ cell-specific transcriptional regulator sohlh2 is essential for early mouse folliculogenesis and oocyte-specific gene expression. Biology of reproduction 2008; 79:1176-1182

29. Rajkovic A, Pangas SA, Ballow D, Suzumori N, Matzuk MM. NOBOX deficiency disrupts early folliculogenesis and oocyte-specific gene expression. Science (New York, NY) 2004; 305:1157-1159

30. Padua MB, Fox SC, Jiang T, Morse DA, Tevosian SG. Simultaneous gene deletion of gata4 and gata6 leads to early disruption of follicular development and germ cell loss in the murine ovary. Biology of reproduction 2014; $91: 24$ 
500 31. Byers M, Kuiper GG, Gustafsson JA, Park-Sarge OK. Estrogen receptor-beta mRNA expression in rat ovary: down-regulation by gonadotropins. Molecular endocrinology (Baltimore, Md) 1997; 11:172-182

32. Slomczynska M, Duda M, Galas J. Estrogen receptor alpha and beta expression in the porcine ovary. Folia histochemica et cytobiologica 2001; 39:137-138

33. Pelletier G, El-Alfy M. Immunocytochemical localization of estrogen receptors alpha and beta in the human reproductive organs. The Journal of clinical endocrinology and

34. Couse JF, Yates MM, Sanford R, Nyska A, Nilson JH, Korach KS. Formation of cystic ovarian follicles associated with elevated luteinizing hormone requires estrogen receptorbeta. Endocrinology 2004; 145:4693-4702

35. Asadi M, Ghafouri-Fard S, Zare-Abdollahi D, Ebrahim-Habibi A, Matin N. Estrogen

36. Lang-Muritano M, Sproll P, Wyss S, Kolly A, Hurlimann R, Konrad D, Biason-Lauber A.

37. Kim JJ, Choi YM, Choung SH, Yoon SH, Lee GH, Moon SY. Estrogen receptor beta gene $+1730 \mathrm{G} /$ A polymorphism in women with polycystic ovary syndrome. Fertility and sterility Estrogen Receptor beta (ESR2). The Journal of clinical endocrinology and metabolism 2010; 93:1942-1947

38. Sundarrajan C, Liao WX, Roy AC, Ng SC. Association between estrogen receptor-beta gene polymorphisms and ovulatory dysfunctions in patients with menstrual disorders. The Journal of clinical endocrinology and metabolism 2001; 86:135-139 
39. Dupont S, Krust A, Gansmuller A, Dierich A, Chambon P, Mark M. Effect of single and compound knockouts of estrogen receptors alpha (ERalpha) and beta (ERbeta) on mouse reproductive phenotypes. Development (Cambridge, England) 2000; 127:4277-4291

40. Couse JF, Yates MM, Deroo BJ, Korach KS. Estrogen receptor-beta is critical to granulosa cell differentiation and the ovulatory response to gonadotropins. Endocrinology 2005; 146:3247-3262

41. Rumi MAK, Singh P, Roby KF, Zhao X, Iqbal K, Ratri A, Lei T, Cui W, Borosha S, Dhakal

42. Britt KL, Saunders PK, McPherson SJ, Misso ML, Simpson ER, Findlay JK. Estrogen

43. Kezele P, Skinner MK. Regulation of ovarian primordial follicle assembly and development by estrogen and progesterone: endocrine model of follicle assembly. Endocrinology 2003; 144:3329-3337

44. Rumi MA, Dhakal P, Kubota K, Chakraborty D, Lei T, Larson MA, Wolfe MW, Roby KF,

45. Chakravarthi VP, Khristi V, Ghosh S, Yerrathota S, Dai E, Roby KF, Wolfe MW, Rumi 
46. Compton DR, Sheng S, Carlson KE, Rebacz NA, Lee IY, Katzenellenbogen BS, Katzenellenbogen JA. Pyrazolo[1,5-a]pyrimidines: estrogen receptor ligands possessing estrogen receptor beta antagonist activity. Journal of medicinal chemistry 2004; 47:58725893

47. Meyers MJ, Sun J, Carlson KE, Marriner GA, Katzenellenbogen BS, Katzenellenbogen

JA. Estrogen receptor-beta potency-selective ligands: structure-activity relationship studies of diarylpropionitriles and their acetylene and polar analogues. Journal of medicinal

48. Terranova PF, Garza F. Relationship between the preovulatory luteinizing hormone (LH) surge and androstenedione synthesis of preantral follicles in the cyclic hamster: detection

49. Roby KF, Son DS, Terranova PF. Alterations of events related to ovarian function in tumor

50. Roby KF. Alterations in follicle development, steroidogenesis, and gonadotropin receptor binding in a model of ovulatory blockade. Endocrinology 2001; 142:2328-2335

51. Roby KF, Son DS, Taylor CC, Montgomery-Rice V, Kirchoff J, Tang S, Terranova PF. Alterations in reproductive function in SRC tyrosine kinase knockout mice. Endocrine

52. Pedersen T, Peters H. Proposal for a classification of oocytes and follicles in the mouse ovary. Journal of reproduction and fertility 1968; 17:555-557

53. Reddy P, Zheng W, Liu K. Mechanisms maintaining the dormancy and survival of 
54. Wang C, Roy SK. Development of primordial follicles in the hamster: role of estradiol17beta. Endocrinology 2007; 148:1707-1716

55. Zachos NC, Billiar RB, Albrecht ED, Pepe GJ. Developmental regulation of baboon fetal ovarian maturation by estrogen. Biology of reproduction 2002; 67:1148-1156

56. de Vet A, Laven JS, de Jong FH, Themmen AP, Fauser BC. Antimullerian hormone serum levels: a putative marker for ovarian aging. Fertility and sterility 2002; 77:357-362

57. Meduri G, Massin N, Guibourdenche J, Bachelot A, Fiori O, Kuttenn F, Misrahi M,

Touraine P. Serum anti-Mullerian hormone expression in women with premature ovarian

58. Swedenborg E, Pongratz I, Gustafsson JA. Endocrine disruptors targeting ERbeta function. International journal of andrology 2010; 33:288-297

59. Patel S, Zhou C, Rattan S, Flaws JA. Effects of Endocrine-Disrupting Chemicals on the

60. Zhang L, Sedykh A, Tripathi A, Zhu H, Afantitis A, Mouchlis VD, Melagraki G, Rusyn I,

Tropsha A. Identification of putative estrogen receptor-mediated endocrine disrupting chemicals using QSAR- and structure-based virtual screening approaches. Toxicology and

61. Shanle $\mathrm{EK}, \mathrm{Xu} \mathrm{W}$. Endocrine disrupting chemicals targeting estrogen receptor signaling: identification and mechanisms of action. Chemical research in toxicology 2011; 24:6-19 Hamalainen T, Peng SL, Lan ZJ, Cooney AJ, Huhtaniemi I, Liu K. Oocyte-specific deletion of Pten causes premature activation of the primordial follicle pool. Science (New York, NY) 2008; 319:611-613 
63. Edson MA, Nagaraja AK, Matzuk MM. The mammalian ovary from genesis to revelation. Endocrine reviews 2009; 30:624-712

64. Zhao Y, Zhang Y, Li J, Zheng N, Xu X, Yang J, Xia G, Zhang M. MAPK3/1 participates in the activation of primordial follicles through mTORC1-KITL signaling. J Cell Physiol $2018 ; 233: 226-237$

65. Blume-Jensen $\mathrm{P}$, Janknecht $\mathrm{R}$, Hunter $\mathrm{T}$. The kit receptor promotes cell survival via activation of PI 3-kinase and subsequent Akt-mediated phosphorylation of Bad on Ser136. Current biology : CB 1998; 8:779-782

66. Jin X, Han CS, Yu FQ, Wei P, Hu ZY, Liu YX. Anti-apoptotic action of stem cell factor on oocytes in primordial follicles and its signal transduction. Molecular reproduction and development 2005; 70:82-90

67. John GB, Shidler MJ, Besmer P, Castrillon DH. Kit signaling via PI3K promotes ovarian

68. Zhao L, Du X, Huang K, Zhang T, Teng Z, Niu W, Wang C, Xia G. Rac1 modulates the formation of primordial follicles by facilitating STAT3-directed Jagged1, GDF9 and

69. Jadrich JL, O'Connor MB, Coucouvanis E. Expression of TAK1, a mediator of TGF-beta and BMP signaling, during mouse embryonic development. Gene Expr Patterns 2003;

70. Tsunemoto K, Anzai M, Matsuoka T, Tokoro M, Shin SW, Amano T, Mitani T, Kato H, 
Arrest 1) are required for oocyte-specific gene expression in the mouse. Molecular reproduction and development 2008; 75:1104-1108

616 71. Slupianek A, Nieborowska-Skorska M, Hoser G, Morrione A, Majewski M, Xue L, Morris SW, Wasik MA, Skorski T. Role of phosphatidylinositol 3-kinase-Akt pathway in nucleophosmin/anaplastic lymphoma kinase-mediated lymphomagenesis. Cancer research

72. Bai RY, Ouyang T, Miething C, Morris SW, Peschel C, Duyster J. Nucleophosminanaplastic lymphoma kinase associated with anaplastic large-cell lymphoma activates the phosphatidylinositol 3-kinase/Akt antiapoptotic signaling pathway. Blood 2000; 96:4319-

624 73. Marzec M, Kasprzycka M, Liu X, Raghunath PN, Wlodarski P, Wasik MA. Oncogenic tyrosine kinase NPM/ALK induces activation of the MEK/ERK signaling pathway independently of c-Raf. Oncogene 2007; 26:813-821

74. Tong T, Shen Y, Lee HW, Yu R, Park T. Adenylyl cyclase 3 haploinsufficiency confers 
Table 1. List of antibodies used in the western blot assays

\begin{tabular}{|l|l|l|l|l|l|}
\hline Target & Name of Antibody & Manufacturer, Catalog number & $\begin{array}{l}\text { Species, Mono or } \\
\text { Polyclonal }\end{array}$ & Dilution & RRID \\
\hline ESR2 & Anti-ESR2, clone 68-4 & EMD Millipore, 05-824 P & Rabbit; monoclonal & 5000 & AB_11212759 \\
\hline pAKT & Anti-Phospho-Akt (T308) & Cell Signaling Technology, 13038 & Rabbit; monoclonal & 2000 & AB_2629447 \\
\hline pAKT & Anti-Phospho-Akt (Ser473) & Cell Signaling Technology, 4060 & Rabbit; monoclonal & 2000 & AB_2315049 \\
\hline AKT & Anti- Akt & Cell Signaling Technology, 4691 & Rabbit; monoclonal & 2000 & AB_915783 \\
\hline PTEN & PTEN (D4.3) & Cell Signaling Technology, 9188 & Rabbit; monoclonal & 2000 & AB_2253290 \\
\hline ERK & Anti-p44/42 MAPK (ERK1/2) & Cell Signaling Technology, 4695 & Rabbit; monoclonal & 1000 & AB_390779 \\
\hline pERK & $\begin{array}{l}\text { Anti-Phospho-ERK1-T202/Y204 } \\
+ \text { ERK2-T185/Y187 }\end{array}$ & Cell Signaling Technology, 4370 & Rabbit; monoclonal & 2000 & AB_2315112 \\
\hline pTSC2 & Anti-Phospho-TSC2-T1462 & ABclonal Technology, AP0866 & Rabbit; polyclonal & 2000 & AB_2771631 \\
\hline TSC2 & Anti-TSC2 & ABclonal Technology, A0492 & Rabbit; polyclonal & 2000 & AB_2757221 \\
\hline pmTORC1 & Anti-Phospho-mTOR (Ser2448) & Cell Signaling Technology, 5536 & Rabbit; monoclonal & 2000 & AB_10691552 \\
\hline mTORC1 & Anti-mTOR & Cell Signaling Technology, 2983 & Rabbit; monoclonal & 2000 & AB_2105622 \\
\hline pP70S6K & Anti-pP70S6Kinase (T389) & Cell Signaling Technology, 9234 & Rabbit; monoclonal & 2000 & AB_2269803 \\
\hline \multirow{2}{*}{ pRPS6 } & $\begin{array}{l}\text { Anti-pS6 Ribosomal Protein } \\
\text { (Ser240/244) }\end{array}$ & Cell Signaling Technology,5364 & Rabbit; monoclonal & 2000 & AB_10694233 \\
\hline ACTB & Anti- $\beta$-actin & Sigma-Aldrich (AC15), A 1978 & Mouse; monoclonal & 25,000 & AB_476692 \\
\hline
\end{tabular}


Table 2. List of primers used in the qPCR assays

\begin{tabular}{|l|l|l|l|l|}
\hline Symbol & Reference mRNA & Forward Primer & Reverse Primer & Amplicon \\
\hline Esr2 & NM_012754.1 & 1101F-GAAGCTGAACCACCCAATGT & 1310R-CAGTCCCACCATTAGCACCT & 210bp \\
\hline Kitlg & NM_021843.4 & 296F-CAAAACTGGTGGCGAATCTT & 512R-GCCACGAGGTCATCCACTAT & 217bp \\
\hline Kit & NM_022264.1 & 2768F-GATGCTGATCCCCTGAAAAG & 2962R-AGGAGAGGCTGTGTGGAAGA & 195bp \\
\hline Igf1 & NM_001082477.2 & 155F-CTGGTGGACGCTCTTCAGTT & 320R-TCAGCGGAGCACAGTACATC & 166bp \\
\hline Fgf2 & NM_019305.2 & 697F-CGACCCACACGTCAAACTAC & 770R-CGGTACCTGGCTATGAAGGA & 74bp \\
\hline Ntrk2 & NM_001163168.2 & 1979F-GTGGTGGTGATTGCCTCTGT & 2134R-CCCAGCCTTGTCTTTCCTTT & 156bp \\
\hline Adcyap1r1 & NM_001270579.1 & 378F-TGCCTGTGGCTATTGCTATG & 575R-AGACCTCAGGGCAGCTTACA & 198bp \\
\hline Bmp4 & NM_012927.2 & 539F-GACTTCGAGGCGACACTTCT & 775R-ACTGGTCCCTGGGATGTTCT & 236bp \\
\hline Bmp15 & NM_021670.1 & 525F-GAGGCTGATAAAGCCGTCAG & 633R-AAGTTGATGGCGGTAGACCA & 109bp \\
\hline Gdf9 & NM_021672.1 & 330F-CTACAATACCGTCCGGCTCT & 475R-GCAAGACCGATTTGAGTAAGTG & 146bp \\
\hline Amh & NM_012902.1 & 280F-CTAGCCACCTTCGGAGTCTG & 441R-AGGCTCTTGGAACTTCAGCA & 162bp \\
\hline Bdnf & NM_001270630.1 & 546F-GTCCCTGGCTGACACTTTTG & 733R-CGGCATCCAGGTAATTTTG & 188bp \\
\hline Nobox & NM_001192013.1 & 549F-TCAGAACCGTAGGGCAAAGT & 737R-GGCTCAGTAAAGCCATCCAG & 189bp \\
\hline Sohlh1 & NM_001191852.1 & 437F-CAGACTCCGGGATAGCAAAA & 622R-CCAAGCTGGAGGACTCTGAC & 186bp \\
\hline Gata4 & NM_144730.1 & 1029F-CTCCTACTCCAGCCCCTACC & 1315R-GCCGGTTGATACCATTCATCT & 287bp \\
\hline Npm 2 & NM_203340.1 & 368F-CCTGGGAAGATGATGAGGAA & 612R-CGTCGACCTCTTAGGTTTG & 245bp \\
\hline
\end{tabular}


Figure 1
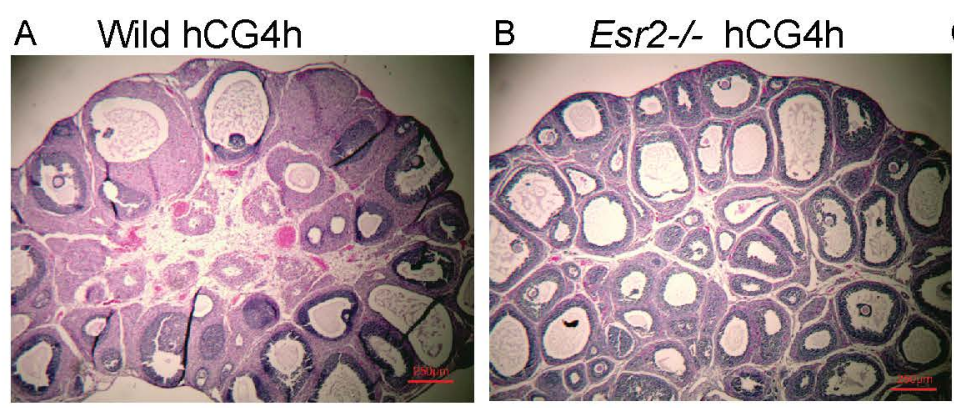

\section{PMSG+hCG4h}

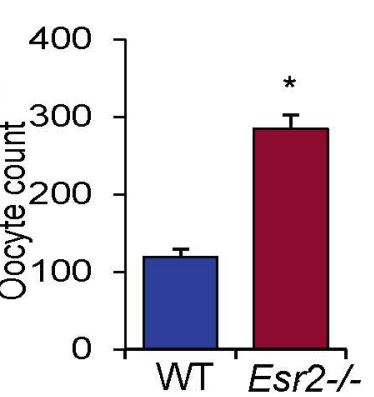

D

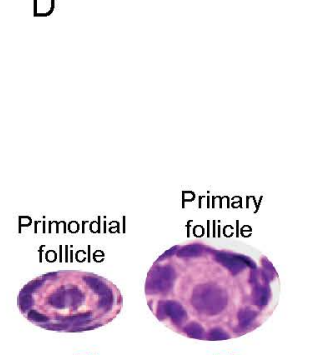

$50 \mu \mathrm{m}$

$50 \mu \mathrm{m}$

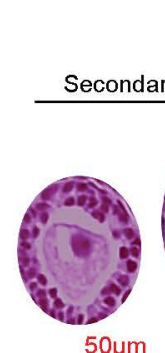

$\underline{50 \mu \mathrm{m}}$

E

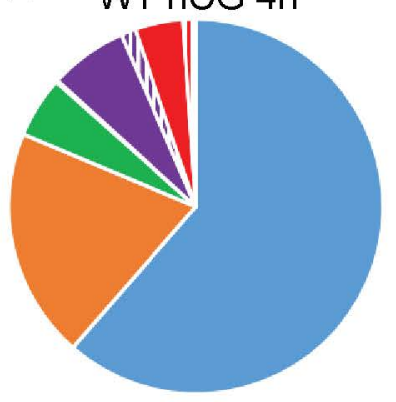

Primordial follicle

Primary follicle
F

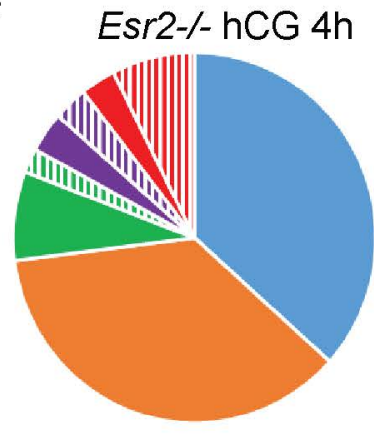

Secondary follicle

- Early antral follicle

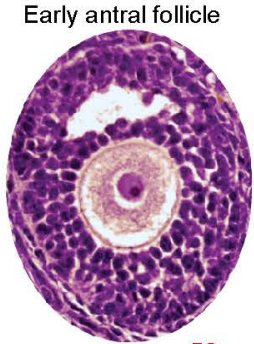

$50 \mu \mathrm{m}$

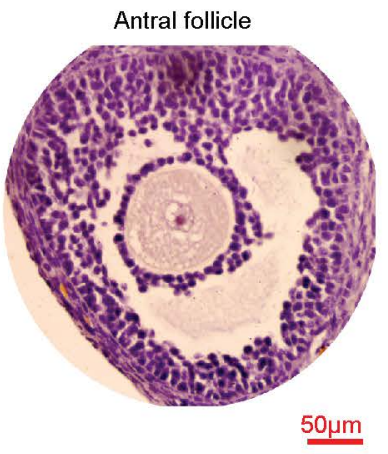

PMSG+hCG4h

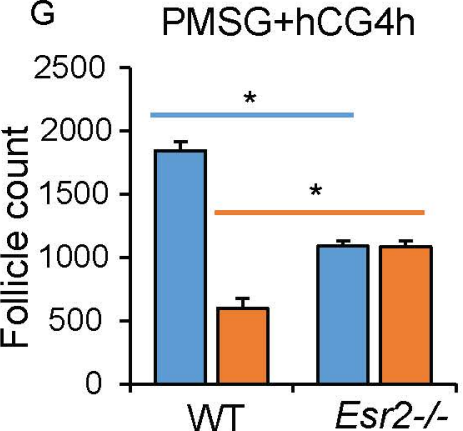

Antral follicle

$\square$ Healthy

III Atretic 
bioRxiv preprint Aqugtres:20i.org/10.1101/2020.02.06.937953; this version posted February 7, 2020. The copyright holder for this preprint (which was not certified by peer review) is the author/funder. All rights reserved. No reuse allowed without permission.
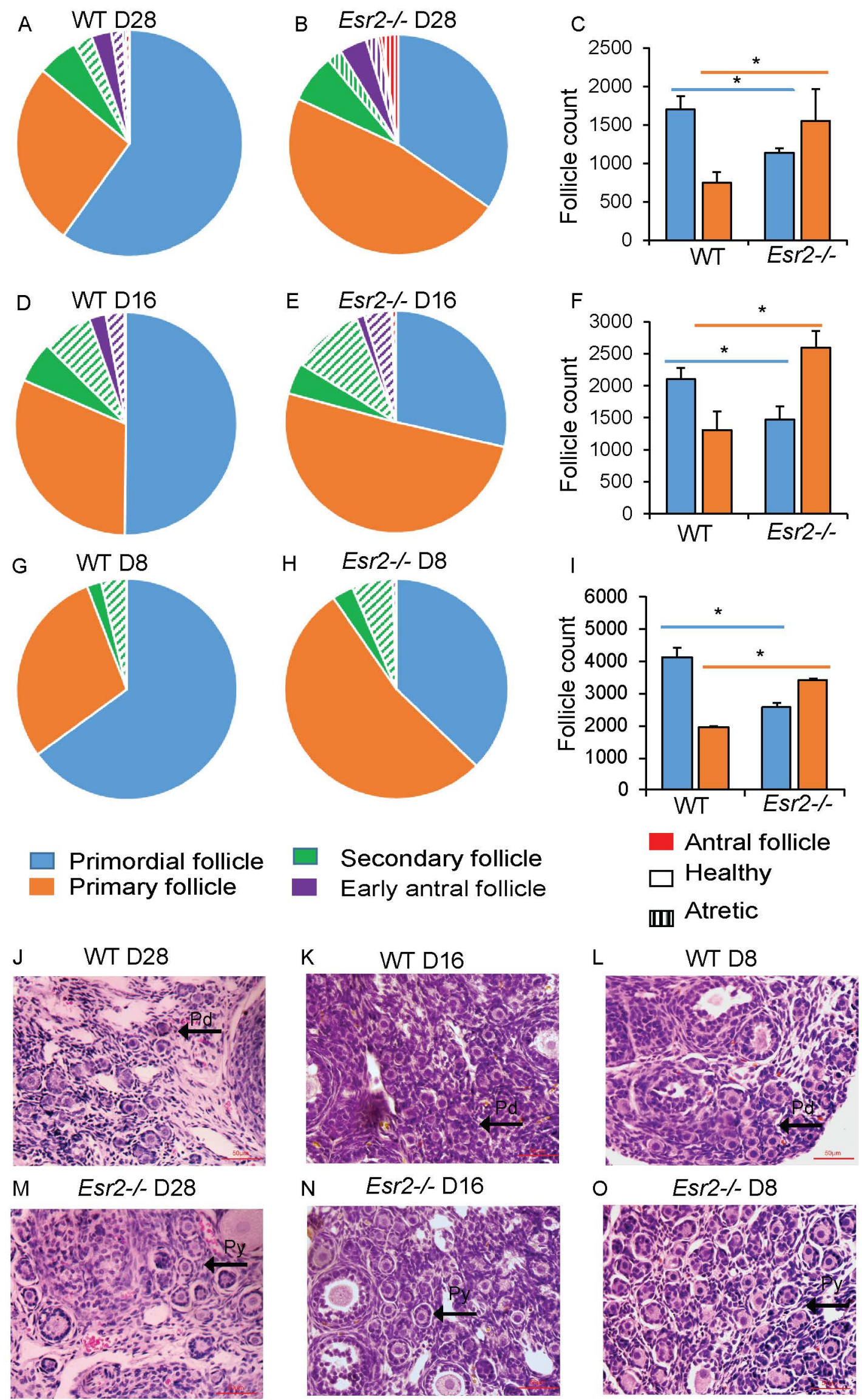
Figure 3

A

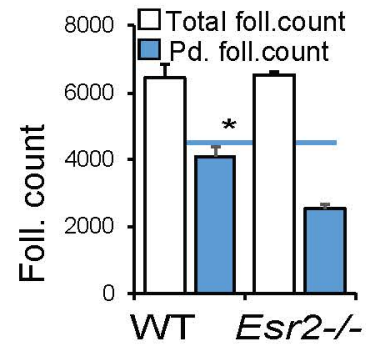

C

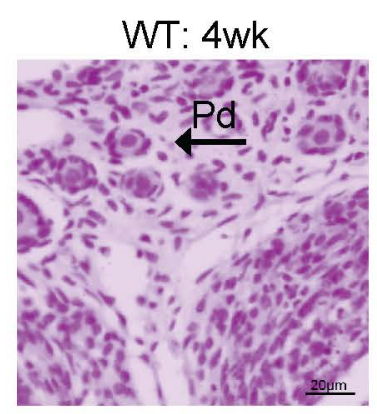

E

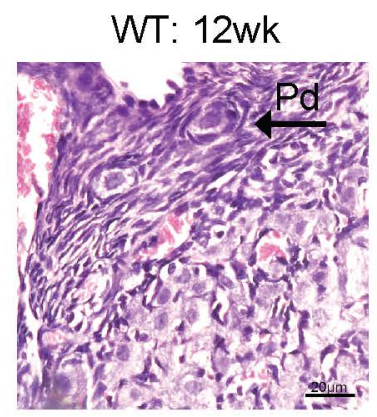

G

WT: 24wk

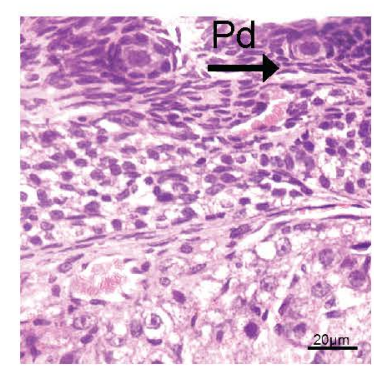

I

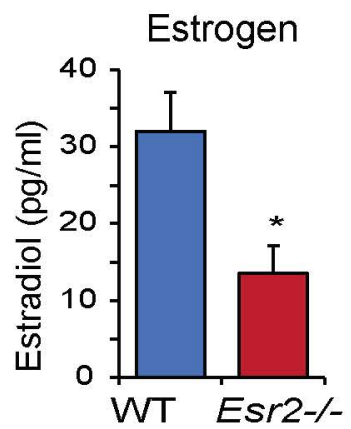

B

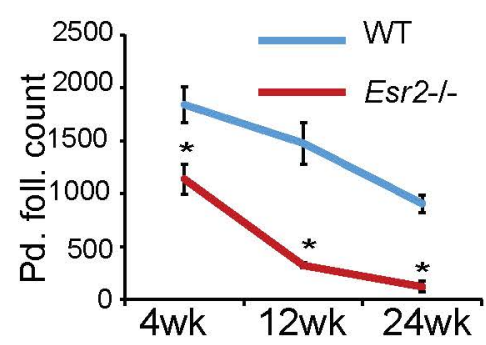

D

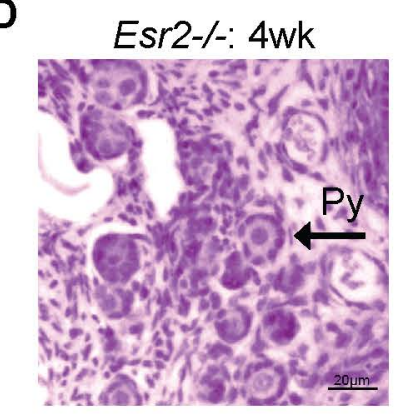

F

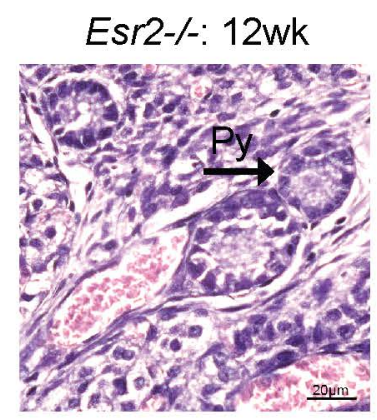

H

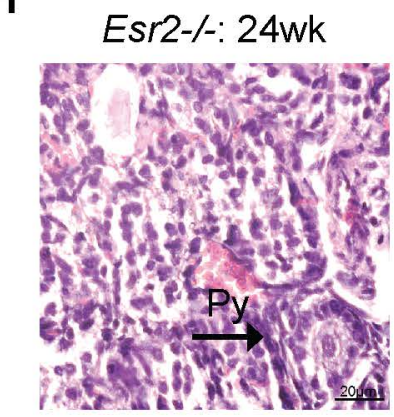

J

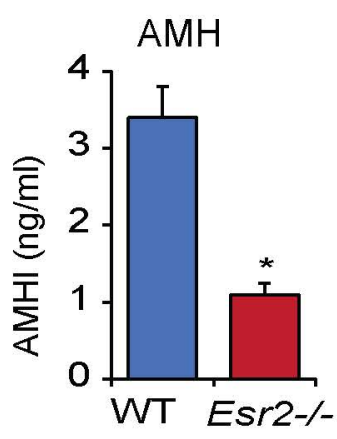


bioRxiv ஈraprintedoizhttps://doi.org/10.1101/2020.02.06.937953; this version posted February 7, 2020. The copyright holder for this preprint (Which was not certified by peer review) is the author/funder. All rights reserved. No reuse allowed without permission.

A

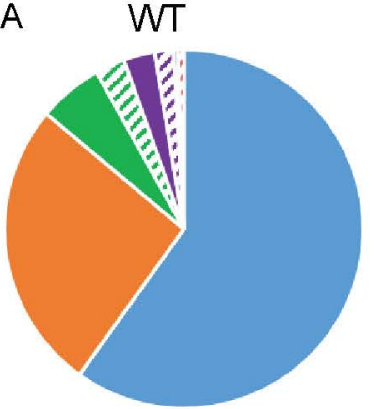

D Control

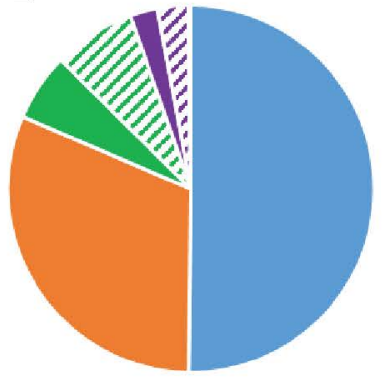

G

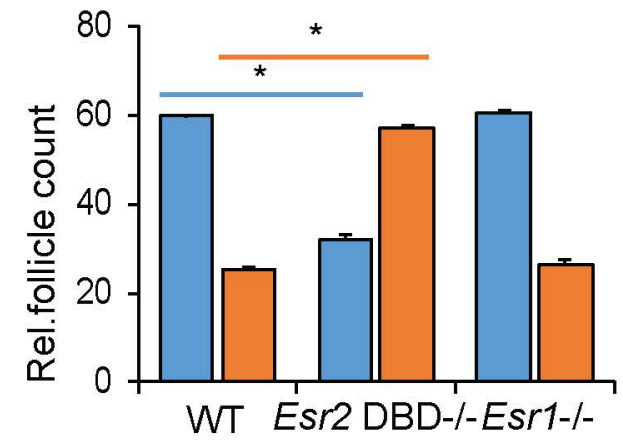

B Esr2 DBD-/-

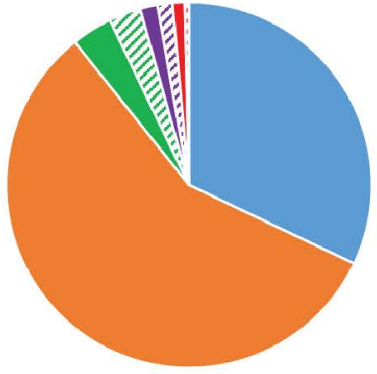

E

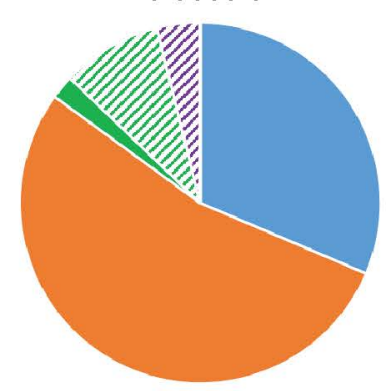

C

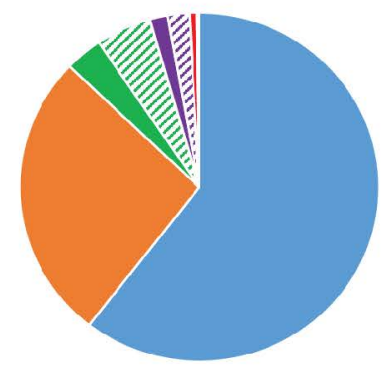

F

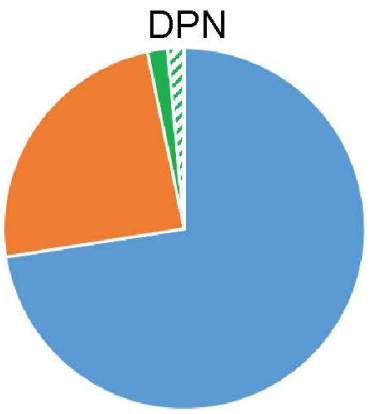

$\mathrm{H}$

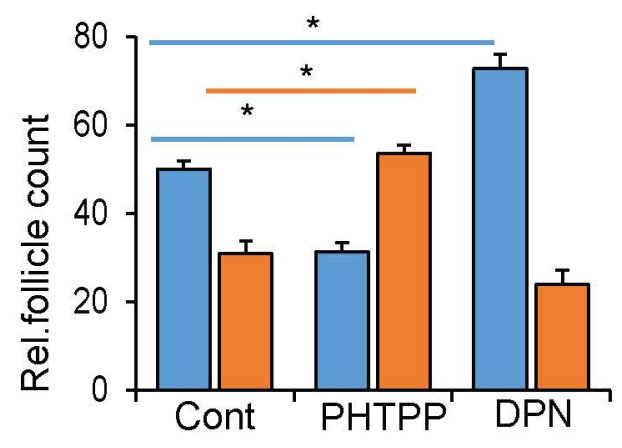

$\square$ Primordial follicle Primary follicle
Secondary follicle

Early antral follicle
Antral follicle $\square$ Healthy IIII Atretic
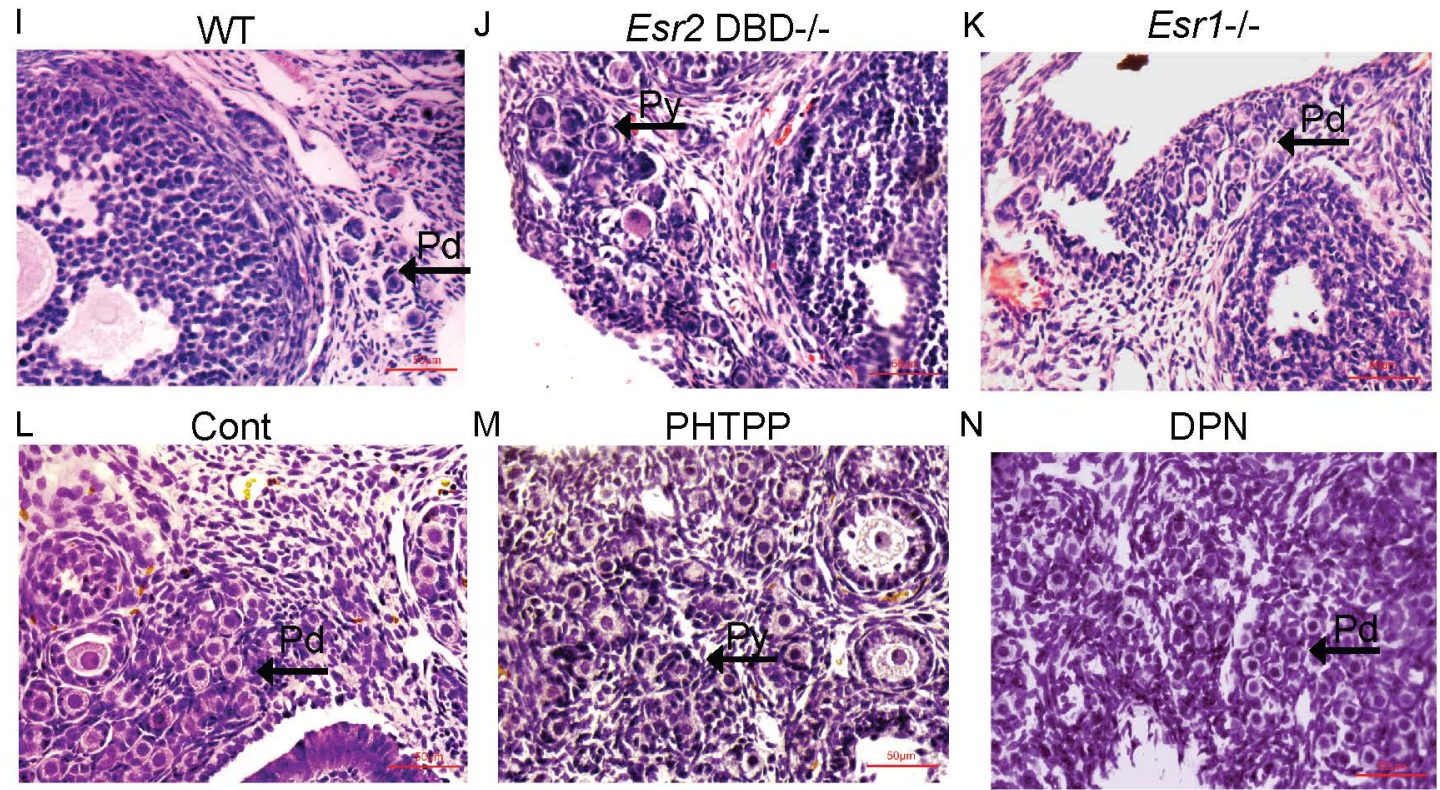

$M$

PHTPP

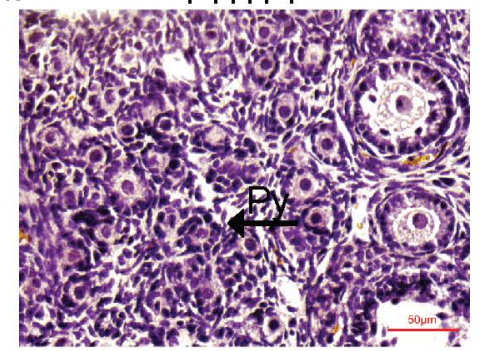

$\mathrm{N}$

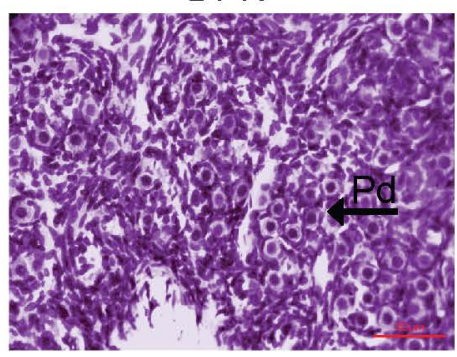


A

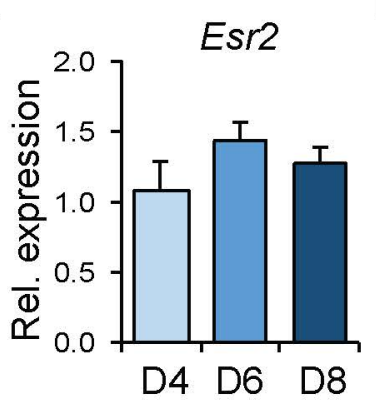

$\mathbf{F}$

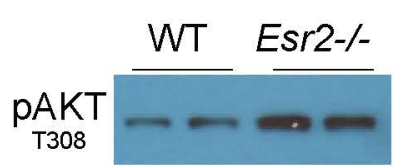

AKT

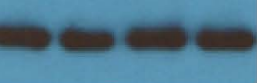

$J$

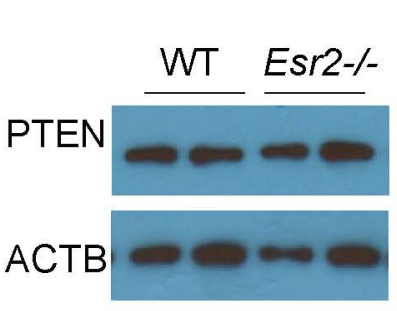

N

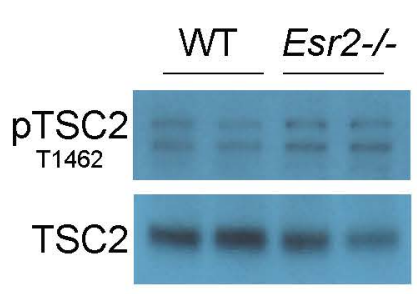

$\mathbf{R}$

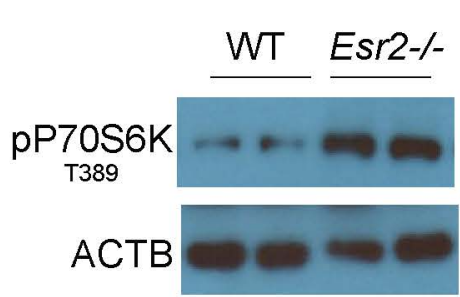

B

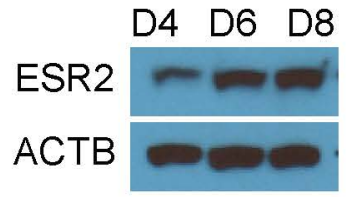

G

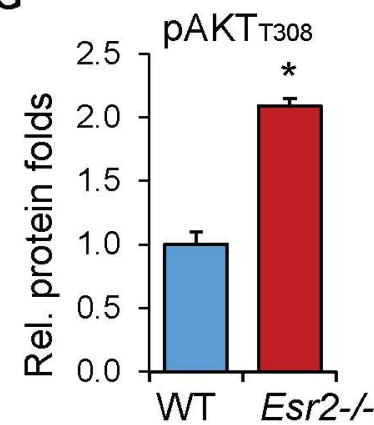

K

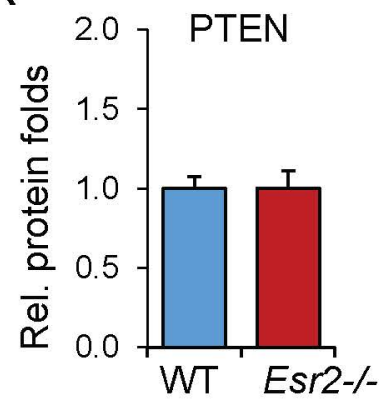

$\mathbf{P}$
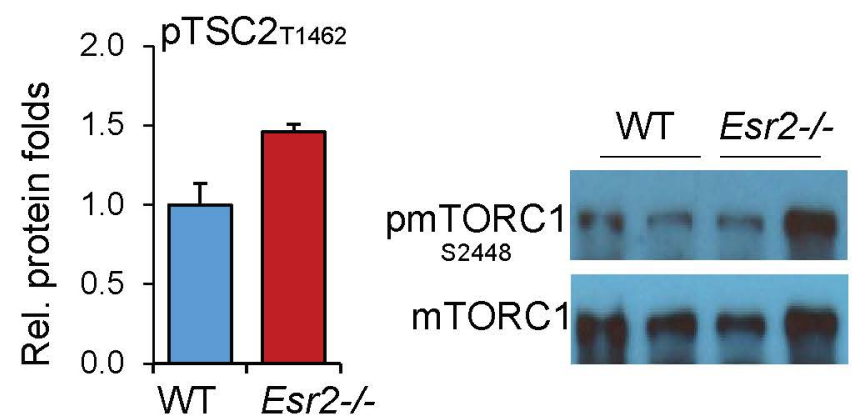

S

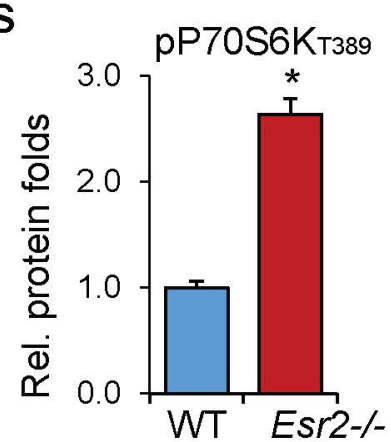

L

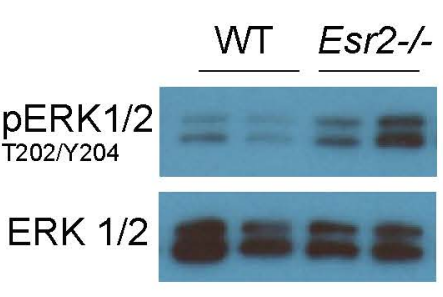

D

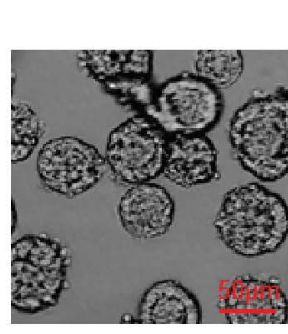

H

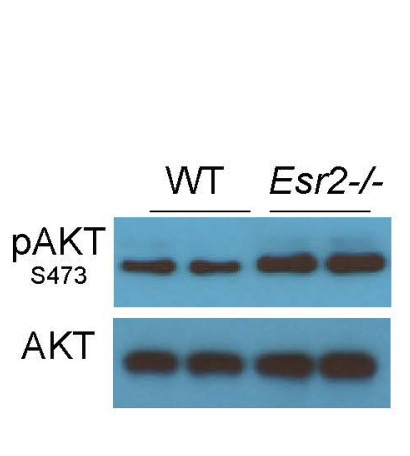

I

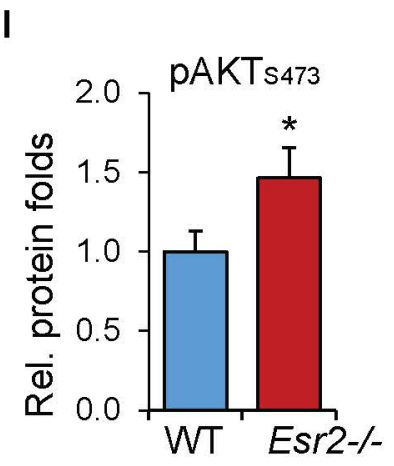

M

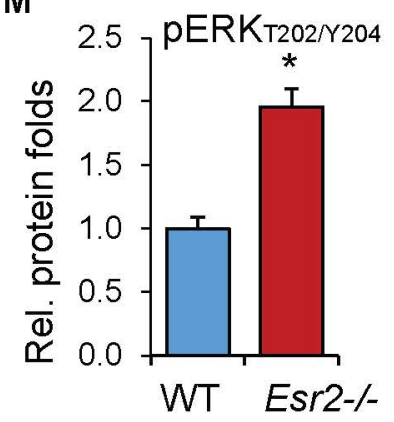

Q

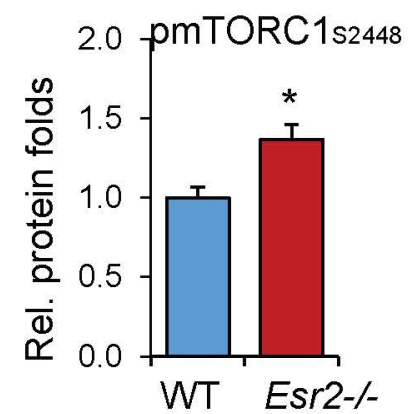

U
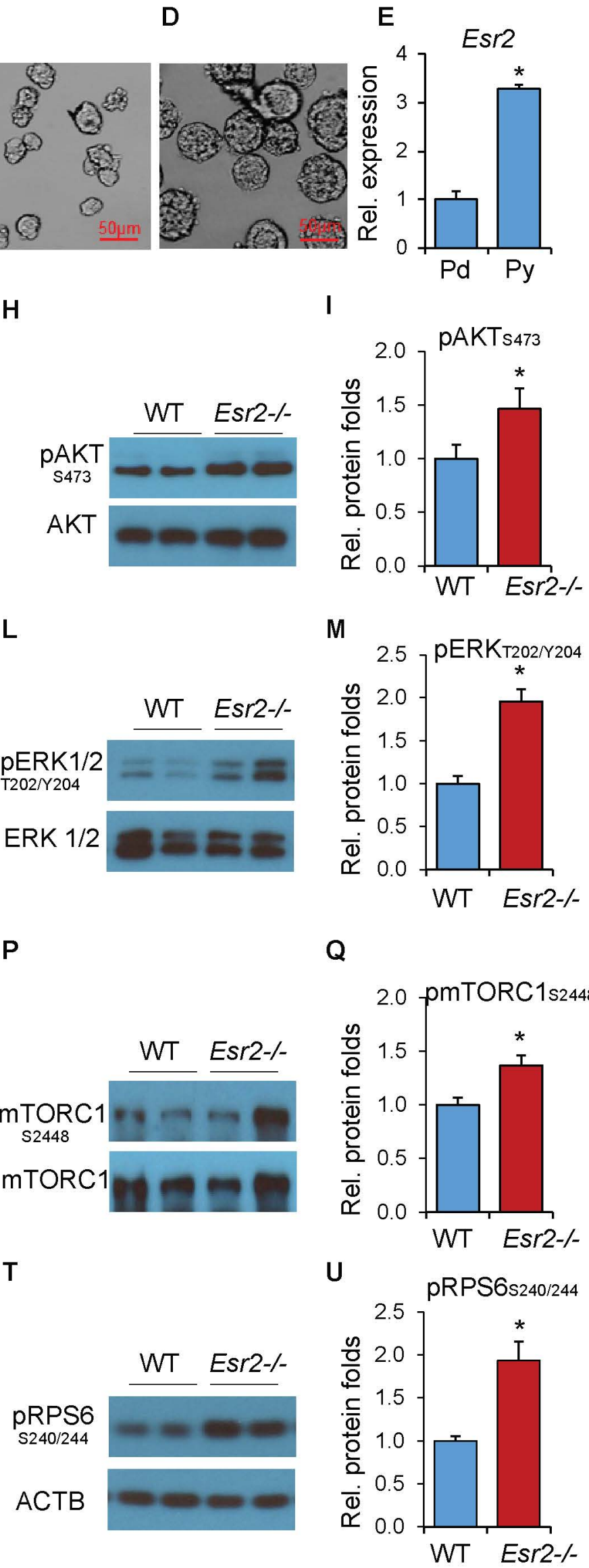


\section{Figure 6}
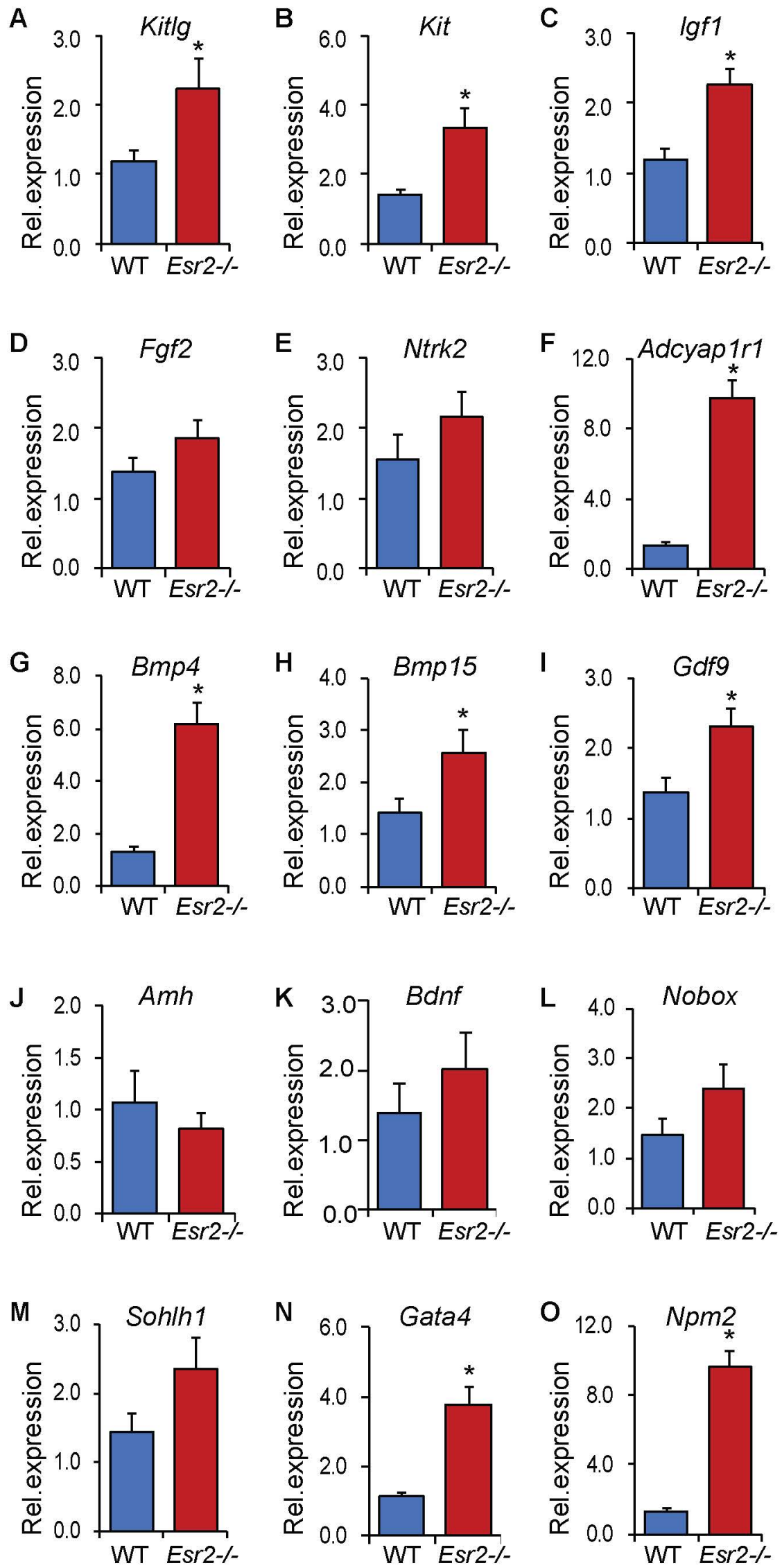


\section{Figure 7}

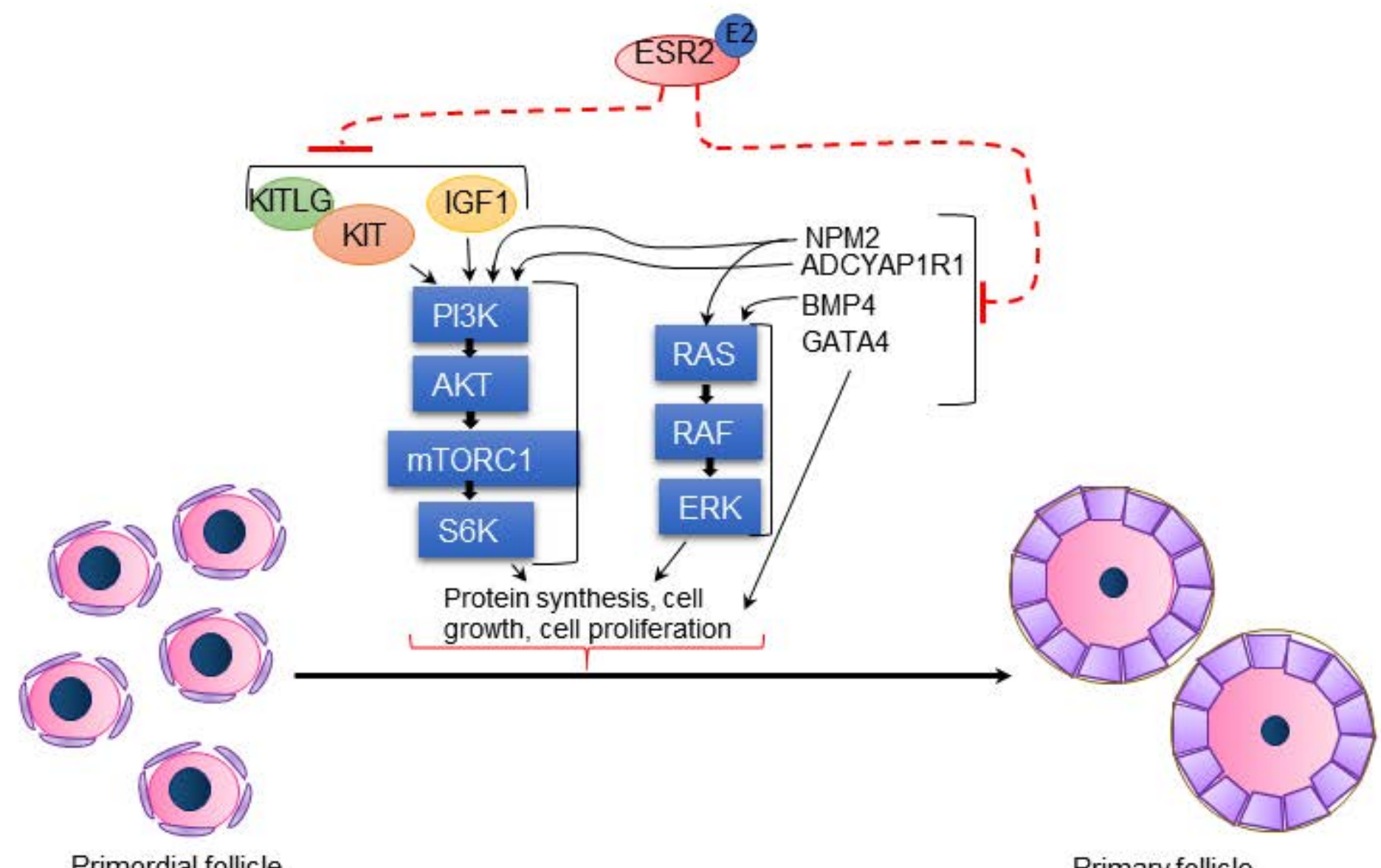

Primordial follicle

Primary follicle 